H.-X. Yi

Nagoya Math. J.

Vol. 138 (1995), 169-177

\title{
A QUESTION OF GROSS AND THE UNIQUENESS OF ENTIRE FUNCTIONS
}

\author{
HONG-XUN YI
}

\section{Introduction and main results}

For any set $S$ and any entire function $f$ let

$$
E_{f}(S)=\bigcup_{a \in S}\{z \mid f(z)-a=0\},
$$

where each zero of $f-a$ with multiplicity $m$ is repeated $m$ times in $E_{f}(S)$ (cf. [1]). It is assumed that the reader is familiar with the notations of the Nevanlinna Theory (see, for example, [2]). It will be convenient to let $E$ denote any set of finite linear measure on $0<r<\infty$, not necessarily the same at each occurrence. We denote by $S(r, f)$ any quantity satisfying $S(r, f)=o(T(r, f))(r \rightarrow \infty, r \notin E)$.

In 1976 Gross proved [3] that there exist three finite sets $S_{j}(j=1,2,3)$, such that any two entire functions $f$ and $g$ satisfying $E_{f}\left(S_{j}\right)=E_{g}\left(S_{j}\right)$ for $j=1,2,3$ must be identical. In the same paper Gross posed the following open question (Question 6): can one find two (or possible even one) finite set $S_{j}(j=1,2)$ such that any two entire functions $f$ and $g$ satisfying $E_{f}\left(S_{j}\right)=$ $E_{g}\left(S_{j}\right)(j=1,2)$ must be identical ?

The present author [4] proved the following result which is partial answer of the above question.

THeorem A. Let $S_{1}=\left\{w \mid(w-a)^{n}-b^{n}=0\right\}, S_{2}=\{c\}$, where $n>4, a, b$ and $c$ are constants such that $b \neq 0, c \neq a$ and $(c-a)^{2 n} \neq b^{2 n}$. Suppose that $f$ and $g$ are nonconstant entire functions satisfying $E_{f}\left(S_{\jmath}\right)=E_{g}\left(S_{j}\right)$ for $j=1,2$. Then $f \equiv g$.

The set $S$ such that for any two nonconstant entire funstions $f$ and $g$ the condition $E_{f}(S)=E_{g}(S)$ implies $f \equiv g$ is called a unique range set (URS in brief) of

Received April 22, 1994.

Revised July 14, 1994.

This research was partially supported by the National Natural Science Fundation of China. 
entire functions (cf. [5]). In 1982, F. Gross and C. C. Yang proved the following result.

THEOREM B [5]. The set $S=\left\{w \mid e^{w}+w=0\right\}$ is a URS of entire functions.

Note that the set $S=\left\{w \mid e^{w}+w=0\right\}$ contains infinite number of elements and so Theorem B does not answer the question posed by Gross.

In this paper we give a positive answer to Gross's question. In fact, we prove more generally the following theorem.

THEOREM 1. Let $n$ and $m$ be two positive integers such that $n$ and $m$ have no common factor and $n>2 m+4$. Let $a$ and $b$ be two nonzero constants such that the algebraic equation $w^{n}+a w^{n-m}+b=0$ has no multiple roots. Then the set $S=$ $\left\{w \mid w^{n}+a w^{n-m}+b=0\right\}$ is a URS of entire functions.

EXAmple. The set $S=\left\{w \mid w^{7}+w^{6}+1=0\right\}$ is a URS of entire functions with 7 elements.

Now it is natural to ask the following question:

Can one find a URS of entire functions with less than 7 elements?

Now we introduce the following notations:

$$
\begin{aligned}
& U_{E}=\{S \mid S \text { is a URS of entire functions }\}, \\
& C_{E}=\min \left\{n(S) \mid S \in U_{E}\right\},
\end{aligned}
$$

where $n(S)$ denotes the cardinal number of the set $S$.

The above example shows that $C_{E} \leqslant 7$. In this paper we prove the following result.

THEOREM 2. $C_{E} \geqslant 4$.

\section{Some lemmas}

The following lemmas will be needed in the proof of Theorem 1 .

Lemma 1 (see [6]). Let $f$ and $g$ be two nonconstant meromorphic functions, and let $c_{1}, c_{2}$ and $c_{3}$ be three nonzero constants. If

$$
c_{1} f+c_{2} g=c_{3},
$$

then 


$$
T(r, f)<\bar{N}\left(r, \frac{1}{f}\right)+\bar{N}\left(r, \frac{1}{g}\right)+\bar{N}(r, f)+S(r, f)
$$

Lemma 2 (see [7]). Let $f_{1}, f_{2}, \ldots, f_{n}$ be linearly independent meromorphic functions satisfying

$$
\sum_{j=1}^{n} f_{j}=1
$$

Then for $k=1,2, \ldots, n$ we have

$$
\begin{gathered}
T\left(r, f_{k}\right)<\sum_{j=1}^{n} N\left(r, \frac{1}{f_{j}}\right)+N\left(r, f_{k}\right)+N(r, D)-\sum_{j=1}^{n} N\left(r, f_{j}\right) \\
-N\left(r, \frac{1}{D}\right)+o(T(r))(r \notin E),
\end{gathered}
$$

where $D$ denotes the Wronskian of the functions $f_{1}, f_{2}, \ldots, f_{n}$, and $T(r)$ denotes the maximum of $T\left(r, f_{j}\right), j=1,2, \ldots, n$.

Lemma 3 (see [8]). Let $f_{1}, f_{2}(\not \equiv 0)$ and $f_{3}$ be three meromorphic functions satisfying $f_{1}+f_{2}+f_{3}=1$, and let $g_{1}=-f_{3} / f_{2}, g_{2}=1 / f_{2}$ and $g_{3}=-f_{1} / f_{2}$. If $f_{1}, f_{2}$ and $f_{3}$ are linearly independent, then $g_{1}, g_{2}$ and $g_{3}$ are linearly independent.

Lemma 4 (see [9]). Let $f$ be a nonconstant meromorphic function, and let $P(f)$ be a polynomial in $f$ of the form

$$
P(f)=a_{0} f^{n}+a_{1} f^{n-1}+\cdots+a_{n-1} f+a_{n},
$$

where $a_{0}(\neq 0), a_{1}, \ldots, a_{n}$ are constants. Then

$$
T(r, P(f))=n T(r, f)+S(r, f) .
$$

\section{Proof of Theorem 1}

Let $w_{1}, w_{2}, \ldots, w_{n}$ be the roots of equation $w^{n}+a w^{n-m}+b=0$. Suppose that $f$ and $g$ are nonconstant entire functions satisfying $E_{f}(S)=E_{g}(S)$. From Nevanlinna's second fundamental theorem, we have

$$
\begin{aligned}
(n-1) T(r, g) & <\sum_{j=1}^{n} N\left(r, \frac{1}{g-w_{j}}\right)+S(r, g) \\
& =\sum_{j=1}^{n} N\left(r, \frac{1}{f-w_{j}}\right)+S(r, g)
\end{aligned}
$$




$$
<n T(r, f)+S(r, g)
$$

Thus

$$
T(r, g)=0(T(r, f)) \quad(r \notin E) .
$$

Again by $E_{f}(S)=E_{g}(S)$, we obtain

$$
\frac{f^{n}+a f^{n-m}+b}{g^{n}+a g^{n-m}+b}=e^{n},
$$

where $h$ is an entire function. From Lemma $4,(1)$ and (3), we have

$$
\begin{aligned}
T\left(r, e^{h}\right) & <T\left(r, f^{n}+a f^{n-m}+b\right)+T\left(r, g^{n}+a g^{n-m}+b\right)+0(1) \\
& =n T(r, f)+n T(r, g)+S(r, f) \\
& <\frac{n(2 n-1)}{n-1} \cdot T(r, f)+S(r, f) .
\end{aligned}
$$

Thus

$$
T\left(r, e^{h}\right)=0(T(r, f)) \quad(r \notin E)
$$

Let us put

$$
f_{1}=-\frac{1}{b} f^{n-m}\left(f^{m}+a\right)
$$

$$
f_{2}=e^{h}
$$

$$
f_{3}=\frac{1}{b} g^{n-m}\left(g^{m}+a\right) e^{n} \text {, }
$$

and $T(r)$ denote the maximum of $T(r, f), j=1,2,3$. From (3), (5), (6) and (7), we obtain

$$
f_{1}+f_{2}+f_{3}=1
$$

From (2), (4), (5), (6) and (7), we have

$$
T(r)=0(T(r, f))(r \notin E) .
$$

Suppose that $f_{1}, f_{2}$ and $f_{3}$ are linearly independent. Applying Lemma 2 to the functions $f_{j}(j=1,2,3)$, from (8) and (9) we have

$$
T\left(r, f_{1}\right)<\sum_{j=1}^{3} N\left(r, \frac{1}{f_{j}}\right)-N\left(r, \frac{1}{D}\right)+o(T(r, f))(r \notin E),
$$


where

$$
D=\left|\begin{array}{lll}
f_{1} & f_{2} & f_{3} \\
f_{1}^{\prime} & f_{2}^{\prime} & f_{3}^{\prime} \\
f_{1}^{\prime \prime} & f_{2}^{\prime \prime} & f_{3}^{\prime \prime}
\end{array}\right|
$$

From (5), (6) and (7), we have

$$
\begin{aligned}
\sum_{j=1}^{3} N\left(r, \frac{1}{f_{j}}\right) & =(n-m) N\left(r, \frac{1}{f}\right)+N\left(r, \frac{1}{f^{m}+a}\right) \\
& +(n-m) N\left(r, \frac{1}{g}\right)+N\left(r, \frac{1}{g^{m}+a}\right) .
\end{aligned}
$$

By looking at the zeros of $f$ and $g$, from (5), (6), (7) and (11) we see that

$$
N\left(r, \frac{1}{D}\right) \geqslant(n-m) N\left(r, \frac{1}{f}\right)-2 \bar{N}\left(r, \frac{1}{f}\right)+(n-m) N\left(r, \frac{1}{g}\right)-2 \bar{N}\left(r, \frac{1}{g}\right)
$$

From Lemma 4, (5), (10), (12) and (13), we deduce

$$
\text { (14) } \begin{aligned}
n T(r, f) & <2 \bar{N}\left(r, \frac{1}{f}\right)+N\left(r, \frac{1}{f^{m}+a}\right)+2 \bar{N}\left(r, \frac{1}{g}\right)+N\left(r, \frac{1}{g^{m}+a}\right)+o(T(r, f)) \\
& <2 T(r, f)+T\left(r, f^{m}+a\right)+2 T(r, g)+T\left(r, g^{m}+a\right)+o(T(r, f)) \\
= & (2+m) T(r, f)+(2+m) T(r, g)+o(T(r, f))(r \notin E) .
\end{aligned}
$$

Let $g_{1}=-f_{3} / f_{2}=-\frac{1}{b} g^{n-m}\left(g^{m}+a\right), g_{2}=1 / f_{2}=e^{-h}$ and $g_{3}=-f_{1} / f_{2}=$ $\frac{1}{b} f^{n-m}\left(f^{m}+a\right) e^{-n}$. From (8) we obtain

$$
g_{1}+g_{2}+g_{3}=1 \text {. }
$$

By Lemma 3 we know that $g_{1}, g_{2}$ and $g_{3}$ are linearly independent. In the same manner as above, we have

(15) $n T(r, g)<(2+m) T(r, g)+(2+m) T(r, f)+o(T(r, f))(r \notin E)$.

Combining (14) and (15) we get

$$
(n-2 m-4)(T(r, f)+T(r, g))<o(T(r, f))(r \notin E) .
$$

Since $n>2 m+4$, (16) is absurd. Hence $f_{1}, f_{2}$ and $f_{3}$ are linearly dependent. Then, there exist three constants $\left(c_{1}, c_{2}, c_{3}\right) \neq(0,0,0)$ such that

$$
c_{1} f_{1}+c_{2} f_{2}+c_{3} f_{3}=0 \text {. }
$$


If $c_{1}=0$, from (17) we have $c_{2} \neq 0, c_{3} \neq 0$ and

$$
f_{3}=-\frac{c_{2}}{c_{3}} f_{2} \text {. }
$$

Hence, from (6) and (7) we obtain

$$
g^{n}+a g^{n-m}=-b c_{2} / c_{3},
$$

which is impossible. Thus $c_{1} \neq 0$ and

$$
f_{1}=-\frac{c_{2}}{c_{1}} f_{2}-\frac{c_{3}}{c_{1}} f_{3}
$$

Now combining (8) and (18) we get

$$
\left(1-\frac{c_{2}}{c_{1}}\right) f_{2}+\left(1-\frac{c_{3}}{c_{1}}\right) f_{3}=1
$$

We discuss the following three cases.

(a) Assume $c_{1} \neq c_{2}$ and $c_{1} \neq c_{3}$. From (6), (7) and (19) we have

$$
-\frac{1}{b}\left(1-\frac{c_{3}}{c_{1}}\right) g^{n-m}\left(g^{m}+a\right)+e^{-h}=1-\frac{c_{2}}{c_{1}} .
$$

By Lemma 1, Lemma 4 and (20) we obtain

$$
\begin{aligned}
n T(r, g) & <\bar{N}\left(r, \frac{1}{g^{n-m}\left(g^{m}+a\right)}\right)+S(r, g) \\
& =\bar{N}\left(r, \frac{1}{g}\right)+\bar{N}\left(r, \frac{1}{g^{m}+a}\right)+S(r, g) \\
& <(1+m) T(r, g)+S(r, g),
\end{aligned}
$$

which is impossible.

(b) Assume $c_{1}=c_{2}$. From (19) we have $c_{1} \neq c_{3}$ and

$$
f_{3}=\frac{c_{1}}{c_{1}-c_{3}} \text {. }
$$

From (7) and (21) we get

$$
g^{n-m}\left(g^{m}+a\right)=\frac{b c_{1}}{c_{1}-c_{3}} e^{-h}
$$

Let $a_{1}, a_{2}, \ldots, a_{m}$ be the roots of equation $w^{m}+a=0$. From (22) we know that $0, a_{1}, a_{2}, \ldots, a_{m}$ are Picard exceptional values of $g$, which is impossible. 
(c) Assume $c_{1}=c_{3}$. From (19) we have $c_{1} \neq c_{2}$ and

$$
f_{2}=\frac{c_{1}}{c_{1}-c_{2}}
$$

that is

$$
e^{h}=\frac{c_{1}}{c_{1}-c_{2}}
$$

From (5), (7), (8) and (23) we get

$$
-\frac{1}{b} f^{n-m}\left(f^{m}+a\right)+\frac{c_{1}}{b\left(c_{1}-c_{2}\right)} g^{n-m}\left(g^{m}+a\right)=\frac{c_{2}}{c_{2}-c_{1}} .
$$

If $c_{2} \neq 0$, by Lemma 1 and Lemma 4 , we have from (24),

$$
\begin{aligned}
n T(r, f) & <\bar{N}\left(r, \frac{1}{f^{n-m}\left(f^{m}+a\right)}\right)+\bar{N}\left(r, \frac{1}{g^{n-m}\left(g^{m}+a\right)}\right)+S(r, f) \\
& <\bar{N}\left(r, \frac{1}{f}\right)+\bar{N}\left(r, \frac{1}{f^{m}+a}\right)+\bar{N}\left(r, \frac{1}{g}\right)+\bar{N}\left(r, \frac{1}{g^{m}+a}\right)+S(r, f) \\
& <(1+\mathrm{m}) T(r, f)+(1+m) T(r, g)+S(r, f) .
\end{aligned}
$$

In the same manner as above, we have

$$
n T(r, g)<(1+m) T(r, g)+(1+m) T(r, f)+S(r, f) .
$$

Hence,

$$
(n-2 m-2) T(r, f)+(n-2 m-2) T(r, g)<S(r, f),
$$

which is impossible. Thus $c_{2}=0$. From (24) we deduce

$$
f^{n}-g^{n}=-a\left(f^{n-m}-g^{n-m}\right) .
$$

If $f^{n} \not \equiv g^{n}$, from (25) we obtain

$$
\frac{-a \prod_{k=1}^{n-m-1}\left(\frac{f}{g}-v^{k}\right)}{\prod_{j=1}^{n-1}\left(\frac{f}{g}-u^{j}\right)}=g^{m},
$$

where $u=\exp \left(\frac{2 \pi i}{n}\right)$ and $v=\exp \left(\frac{2 \pi i}{n-m}\right)$. From (26) we know that $\frac{f}{g}$ is a nonconstant meromorphic function. Since $n$ and $m$ have no common factors, again from (26) we know that $u^{\prime}(j=1,2, \ldots, n-1)$ are Picard exceptional 
values of $\frac{f}{g}$, which is impossible. Thus $f^{n} \equiv g^{n}$ and $f^{n-m} \equiv g^{n-m}$. However, since $n$ and $m$ have no common factors, we get $f \equiv \mathrm{g}$. This completes the proof of Theorem 1.

\section{Proof of Theorem 2}

Let $S=\left\{a_{1}, a_{2}, a_{3}\right\}$, where $a_{,}(j=1,2,3)$ are any three finite distinct complex numbers. If $a_{2}+a_{3}-2 a_{1}=0$, let

$$
g(z)=2 a_{1}-f(z),
$$

where $f(z)$ is a nonconstant entire function. If $a_{2}+a_{3}-2 a_{1} \neq 0$, let

$$
\begin{aligned}
& f(z)=\frac{\left(a_{2} a_{3}-a_{1}{ }^{2}\right)+\left(a_{2}-a_{1}\right)\left(a_{3}-a_{1}\right) e^{h(z)}}{a_{2}+a_{3}-2 a_{1}}, \\
& g(z)=\frac{\left(a_{2} a_{3}-a_{1}^{2}\right)+\left(a_{2}-a_{1}\right)\left(a_{3}-a_{1}\right) e^{-h(z)}}{a_{2}+a_{3}-2 a_{1}},
\end{aligned}
$$

where $h(z)$ is $a$ nonconstant entire function. It is easy to show that $E_{f}(S)=$ $E_{g}(S)$, but $f \not \equiv g$. Hence $C_{E} \geqslant 4$, which proves Theorem 2 .

\section{REFERENCES}

[1] F. Gross, On the distribution of values of meromorphic functions, Trans. Amer. Math. Soc., 131 (1968), 199-214.

[2] W. K. Hayman, Meromorphic Functions, Clarendon Press, Oxford, 1964.

[3] F. Gross, Factorization of meromorphic functions and some open problems, Com. plex Analysis (Proc. Conf. Univ. Kentucky, Lexington, Ky. 1976), pp. 51-69, Lec. ture Notes in Math. Vol. 599, Springer, Berlin, 1977.

[4] Hong-Xun Yi, Unicity theorems for entire functions, Kodai Math. J., 17 (1994), $133-141$.

[5] F. Gross and C. C. Yang, On preimage range sets of meromorphic functions, Proc. Japan Acad., 58 (1982), 17-20.

[6] Hong-Xun Yi, Meromorphic functions that share three values, Chin. Ann. Math., 9A (1988), 434-440.

[ 7 ] R. Nevanlinna, Le Théorème de Picard-Borel at la Théorie des Fonctions Méromorphes, Gauthier-Villars, Paris, 1929.

[ 8 ] Hong-Xun Yi, Meromorphic functions that share two or three values, Kodai Math J., 13: 3 (1990), 363-372.

[9] C. C. Yang, On deficiencies of differential polynomials II, Math. Z., 125 (1972), $107-112$ 
Department of Mathematics

Shandong University

Jinan, Shandong, 250100

People's Republic of China 